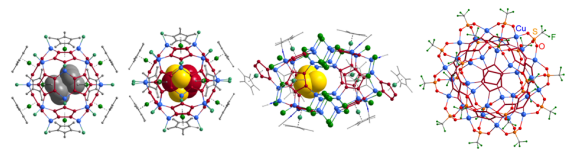

Figure 1. (left to right) Copper haide-based supramolecules encapsulating molecules of ferrocene, $\mathrm{P}_{4} \mathrm{~S}_{3}$, and $\mathrm{As}_{4}$. Inorganic core of a supramolecule based on copper triflate.

Keywords: giant supramolecule, pentaphosphaferrocene, inclusion compound, host-guest interaction, intermolecular interaction, single-crystal X-ray diffraction

\section{MS33-P7 Small Angle Neutron Diffraction on the Vortex Lattice of Type II Superconductors}

Jorge L. Gavilano ${ }^{1}$, Jonathan White ${ }^{2}$, Simon Gerber ${ }^{1}$, Nikola Galvan $^{2}$, B. Biswas ${ }^{3}$, Daniel Mazzone ${ }^{2}$, Edwards Forgan ${ }^{4}$, Hazuki Kawano-Furukawa ${ }^{5}$

1. SwissFEL Photonic Group, Paul Scherrer Institut, Villigen PSI, Switzerland

2. Laboratory for Neutron Scattering and Imaging, Paul Scherrer Institut, Villigen PSI, Switzerland

3. Physics Department, University of Warwick, Coventry, United Kingdom

4. School of Physics and Astronomy, University of Birmingham, United Kingdom

5. Division of Natural/Applied Science, Ochanomizu University, Bunkyo-ku, Tokyo, Japan

email: jorge.gavilano@psi.ch

In type II superconductors in magnetic fields $H_{\mathrm{cl}}<H<\mathrm{H}_{\mathrm{c} 2}$ the flux form a Vortex Lattice VL, whose geometry represents a delicate balance of the electronic Fermi surface features and pinning. This provides a very sensitive probe of some microscopic electronic properties which can be measured using small angle neutron scattering technique SANS. Below we describe selected results.

i) $\mathrm{YBa}_{2} \mathrm{Cu}_{3} \mathrm{O}_{7}$ with $\delta=0,0.15$ at $2 \mathrm{~K}$ and for fields of up to $16 \mathrm{~T}^{3}$ applied parallel to the crystal c-axis, we observe in the SANS data (see Fig. 1) a sequence of field-driven and first-order transitions between different VL structures. By rotating the field away from the c-axis, we observe each structure transition to shift to either higher or lower field dependent on whether the field is rotated towards the [100] or [010] direction. We argue that these transitions are determined by the Fermi Surface Morphology [1].

(ii) $\mathrm{KFe}_{2} \mathrm{As}_{2}$, and related materials. We find an intrinsic anisotropy of the superconducting state in this material. With the SANS technique we monitor the vortex and find a field dependent anisotropy, indicating multiband superconductivity. These results support that $\mathrm{KFe}_{2} \mathrm{As}_{2}$ is Pauli limited for field applied in the basal plane.

iii) The flux-line lattice in CaAlSi has been studied by small-angle neutron scattering. A well-defined hexagonal flux-line lattice is seen just above $\mathrm{Hcl}$ in an applied field of only 54 Oe. A 30_ reorientation of this vortex lattice has been observed in a very low field of $200 \mathrm{Oe}$. This reorientation transition is first-order and reflect nonlocal effects [3].

[1] J of the Phys Soc of Jpn, 84, 44709 (2015), by N Galvan, J White J. Lim et al.

[2] Phys Rev B 93, 104527 (2016) S. Kuhn, H. Kawano-Furukawa, E. Jellyman et al.

[3] Phys. Rev. Lett. 108, 77001 (2012) P.K.Biswas MR Lee B. Balaktishnan et al. 


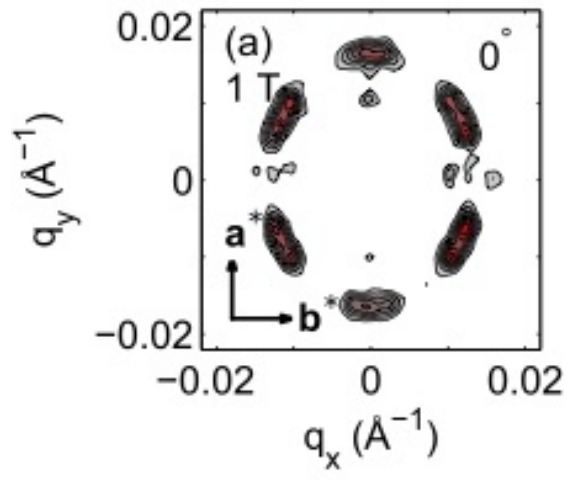

Figure 1. SANS diffraction pattern from a $\mathrm{VL}$ on $\mathrm{YBa}_{2} \mathrm{Cu}_{3} \mathrm{O}_{7-\mathrm{d}}$ at $2 \mathrm{~K}$ and an external field of $1 \mathrm{~T}$ parallel to $\mathrm{c}$

Keywords: SANS, Vortex Lattice, superconductors
MS33-P8 Alien Features: In Superspace, No One Can Hear You Scream...

Amber L. Thompson ${ }^{1}$, Kirsten E. Christensen ${ }^{1}$, Friends from Oxford Chemistry

\section{Chemical Crystallography, Department of Chemistry, Oxford.}

email: amber.thompson@chem.ox.ac.uk

Single-crystal diffraction is the foremost technique used to give the definitive answer to how atoms and molecules pack in the crystalline solid state. As a maturing technique, data collections have been getting faster and faster, and structure determination is becoming more routine, with an increasing number of non-expert users collecting data, solving and refining structures, and publishing their results. However, with the advent of higher intensity laboratory X-ray sources, easier access to synchrotron radiation and more sensitive detectors, more and more molecular structures are showing alien features beyond the realms of conventional crystallography. These include, super-lattice reflections, incommensurate satellite peaks and diffuse features. These can affect everything from the smallest molecules to large macrocycles to frameworks. [1-3]

Here we present a selection of the data that have made us want to scream when working in collaboration with the Chemists in Oxford.

\section{References:}

[1] A. D. Bond, CrystEngComm. (2012), 14, 2363.

[2] A. Schönleber, Z. Kristallogr. (2011), 226, 499.

[3] C. B. Pinheiro, et. al., IUCrJ (2015), 2, 137-154.

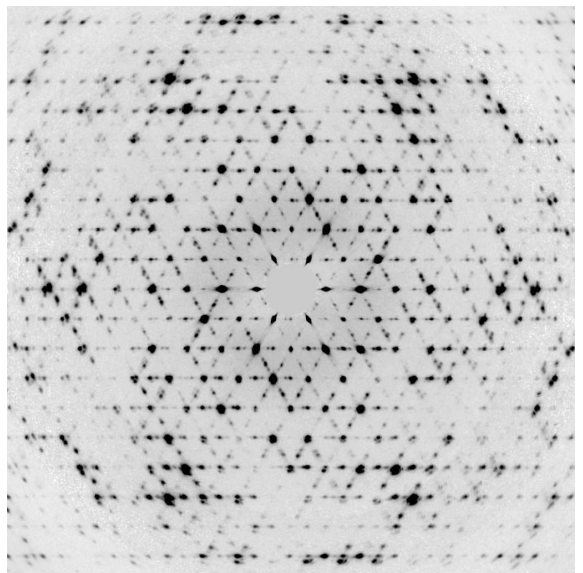

Figure 1. Can you hear us scream?

Keywords: modulated structures, diffuse scattering, non-Bragg 